\title{
Fixed Point Algebras for Easy Quantum Groups
}

\author{
Olivier GABRIEL ${ }^{\dagger}$ and Moritz WEBER ${ }^{\ddagger}$ \\ $\dagger$ University of Copenhagen, Universitetsparken 5, 2100 København Ø, Denmark \\ E-mail: olivier.gabriel.geom@gmail.com \\ URL: http://oliviergabriel.eu \\ $\ddagger$ Fachbereich Mathematik, Universität des Saarlandes, \\ Postfach 151150, 66041 Saabrücken, Germany \\ E-mail: weber@math.uni-sb.de
}

Received June 13, 2016, in final form September 26, 2016; Published online October 01, 2016

http://dx.doi.org/10.3842/SIGMA.2016.097

\begin{abstract}
Compact matrix quantum groups act naturally on Cuntz algebras. The first author isolated certain conditions under which the fixed point algebras under this action are Kirchberg algebras. Hence they are completely determined by their $K$-groups. Building on prior work by the second author, we prove that free easy quantum groups satisfy these conditions and we compute the $K$-groups of their fixed point algebras in a general form. We then turn to examples such as the quantum permutation group $S_{n}^{+}$, the free orthogonal quantum group $O_{n}^{+}$and the quantum reflection groups $H_{n}^{s+}$. Our fixed point-algebra construction provides concrete examples of free actions of free orthogonal easy quantum groups, which are related to Hopf-Galois extensions.
\end{abstract}

Key words: K-theory; Kirchberg algebras; easy quantum groups; noncrossing partitions; fusion rules; free actions; free orthogonal quantum groups; quantum permutation groups; quantum reflection groups

2010 Mathematics Subject Classification: 46L80; 19K99; 81R50

In memory of the late Professor John E. Roberts.

\section{Introduction}

This article was initiated from the meeting of the two authors and their respective interests in easy quantum groups and the fixed point algebra construction. Let us start by reminders on the setting of the present article.

Compact quantum groups (CQGs) were defined by Woronowicz and further studied in a series of papers $[41,42,44]$. Following the paradigm of noncommutative geometry, the general idea is to describe all properties of a compact group $G$ in terms of its algebra $C(G)$ of (continuous) functions, using in particular a comultiplication $\Delta: C(G) \rightarrow C(G \times G) \simeq C(G) \otimes C(G)$ to realise the group law $\mu: G \times G \rightarrow G$. If we then consider (possibly noncommutative) $C^{*}$-algebras with such a comultiplication, we get CQGs as an extension of compact groups. Of course, additional assumptions are needed to make the above rigorous (see Section 2.1 below). To be more precise, we will mainly deal with compact matrix quantum groups (CMQGs).

Among CMQGs, there is a class of particular examples, called easy quantum groups. Categories of partitions and easy quantum groups were first defined by Banica and Speicher in [5] in the orthogonal case. Tarrago and the second author extended their approach to the unitary setting, see [37]. To each easy quantum group is associated a category of partitions, which provides a way to "visualise" it. The basic idea of easy quantum groups is that they should form a tractable sub-class of CMQGs, since they can be described and manipulated via their category of partitions, by a Tannaka-Krein type argument [43]. This line of argument is illustrated by 
the article [18], where Freslon and the second author provided a description of fusion rules for easy quantum groups based on their categories of partitions.

On another note, the Cuntz algebra $\mathcal{O}_{n}$ has been defined as a universal $C^{*}$-algebra by Cuntz in his paper [11] and has evolved over time into one of the most important examples of $C^{*}$-algebras, with applications to classification theory and physics. An example of an application is provided by Doplicher and Roberts's abstract, Tannaka-Krein like duality results in a series of articles (see, e.g., $[15,16])$ for actions of (ordinary) compact groups on $C^{*}$-algebras. This discovery motivated a considerable interest (see for instance [8, 33, 34]). A basic step of Doplicher-Roberts's duality theory is to consider so-called "canonical actions" of compact groups on Cuntz algebras. A source of inspiration for further research in this direction is the article [32], where Pinzari introduces a fixed point algebra $\mathcal{O}_{\lambda(G)}$ from the regular representation $\lambda$ acting on $\mathcal{O}_{L^{2}(G)}$ and proves that given two compact groups $G, G^{\prime}$, the fixed point algebras $\mathcal{O}_{\lambda(G)}$ and $\mathcal{O}_{\lambda\left(G^{\prime}\right)}$ are isomorphic as $\mathbb{Z}$-algebras if and only if $C^{*}(G) \simeq C^{*}\left(G^{\prime}\right)$.

Motivated by the desire of generalising Doplicher-Roberts theory and following the articles $[10,27,31]$, the first author considered an action of a CQG $\mathbb{G}$ on a Cuntz algebra and described its fixed point algebra. More precisely, two conditions (C1) and (C2) were introduced, which ensure that the fixed point algebra is actually a Kirchberg algebra in the UCT class $\mathscr{N}$. Kirchberg-Phillips's classification theory (see $[23,24]$ ) then proves that up to *-isomorphism, the fixed point algebra is characterised by its $K$-theory. In [19], examples of computations of the $K$-theory of the fixed point algebra are given - they only depend on the fusion rules of $\mathbb{G}$.

In the present article, we combine these two directions of research to describe the fixed point algebras of actions of the free orthogonal quantum group $O_{n}^{+}$, the quantum permutation group $S_{n}^{+}$and the quantum reflection group $H_{n}^{s+}$. An interesting feature of the present fixed point algebra construction is that it provides a very concrete realisation of the intertwiner spaces defining the easy quantum group (see Proposition 3.2 below).

The main results of this paper are the reformulation and characterisation of the hypotheses (C1) and (C2) of the fixed point algebra construction theory of [19] in terms of partition categories (see Theorems 3.6 and 3.7 below), together with the identifications of $K$-theory for the fixed point algebras associated to the natural representations of $O_{n}^{+}, S_{n}^{+}$and $H_{n}^{s+}$ (see Theorems 4.1 and 5.11 below). The fixed point algebras for $O_{n}^{+}$and $S_{n}^{+}$are isomorphic while the one for $H_{n}^{s+}$ is very different. Thus, in some sense, the actions of $S_{n}^{+}$and $O_{n}^{+}$are somehow "similar" while $H_{n}^{s+}$ acts very differently. Moreover, since these fixed point algebras depend only on the fusion rules of the CQGs at hand, this phenomenon manifests concretely that the fusion rules of $H_{n}^{s+}$ are very different from those of $O_{n}^{+}$and $S_{n}^{+}$.

This article is organised as follows: in Section 2, we start by a review of the notions of CQGs and CMQGs, before presenting the notions of categories of partitions and easy quantum groups and discussing Cuntz algebras and actions of CMQGs on these. Section 3 is devoted to the fixed point algebra construction properly speaking, while Sections 4 and 5 are detailed studies of examples, namely the free orthogonal quantum group $O_{n}^{+}$and the quantum permutation group $S_{n}^{+}$on the one hand, and the quantum reflection group $H_{n}^{s+}$ on the other hand.

\section{Reminders and review}

\subsection{Compact matrix quantum groups}

In this article, we consider only minimal tensor products of $C^{*}$-algebras. We will deal with compact quantum groups (CQGs) which we denote by $\mathbb{G}$. They are defined by a separable unital $C^{*}$-algebra $C(\mathbb{G})$ together with a unital $*$-algebra homomorphism $\Delta: C(\mathbb{G}) \rightarrow C(\mathbb{G}) \otimes C(\mathbb{G})$ which satisfies coassociativity and cancellation properties - for more details on these objects and their representations, see [41, 44]. 
These compact quantum groups admit (unitary) representations or, equivalently actions on Hilbert spaces and $C^{*}$-algebras. A unitary representation $u$ of $\mathbb{G}$ on a Hilbert space $\mathscr{H}$ of finite dimension $d$ is a $C(\mathbb{G})$-valued $d \times d$ matrix $U=\left(u_{i j}\right) \in M_{d}(C(\mathbb{G}))$ which is unitary and satisfies the coassociativity property

$$
\Delta\left(u_{i j}\right)=\sum_{k} u_{i k} \otimes u_{k j}
$$

In particular, for any $\mathbb{G}$, we have a trivial representation denoted by $\epsilon$, defined by the $(1 \times 1)$ matrix $1 \in C(\mathbb{G})$.

For two fixed representations $u \in M_{d_{1}}(C(\mathbb{G}))$ and $v \in M_{d_{2}}(C(\mathbb{G}))$ acting on the Hilbert spaces $\mathscr{H}_{1}$ and $\mathscr{H}_{2}$, respectively, a linear map $T: \mathscr{H}_{1} \rightarrow \mathscr{H}_{2}$ is an intertwiner (see [41]) if

$$
T u=v T .
$$

We denote by $\operatorname{Hom}(u, v)$ the set of interwiners between $u$ and $v$. If $\operatorname{Hom}(u, v)$ includes an invertible map, then we say that $u$ and $v$ are equivalent. Just like for ordinary compact groups, any representation of $\mathbb{G}$ is equivalent to a unitary one, therefore we will only consider unitary representations and we will refer to these as "representations". A representation $u$ is called irreducible if $\operatorname{Hom}(u, u) \simeq \mathbb{C}$. Representations of CQGs admit notions of direct sum - with the above notations, $u \oplus v$ is a representation on $\mathscr{H}_{1} \oplus \mathscr{H}_{2}-$ and tensor product $-u \otimes v$ is then a representation on $\mathscr{H}_{1} \otimes \mathscr{H}_{2}$.

It is a well-known property of CQGs (see, e.g., [44, Theorem 3.4]) that every unitary representation of a CQG is unitarily equivalent to a direct sum of irreducible unitary representations and any irreducible representation is finite-dimensional. Given two representations $u$ and $v$, we use the notation $u \leqslant v$ to express that the representation $u$ is included in $v$ (i.e., there is an isometry in $\operatorname{Hom}(u, v))$.

Of particular interest for our investigations are compact matrix quantum groups (CMQGs), a particular class of CQGs. A CMQG is given by a so-called fundamental representation $u$, whose coefficients generate a dense subalgebra of $C(\mathbb{G})$. One can define CMQGs in the following way:

Definition 2.1. A compact matrix quantum group is defined by a unital $C^{*}$-algebra $A$ generated by elements $u_{i j}, 1 \leq i, j \leq n$ such that $u=\left(u_{i j}\right)$ and $u^{t}=\left(u_{j i}\right)$ are invertible and the map

$$
\Delta: A \rightarrow A \otimes A, \quad u_{i j} \mapsto \sum_{k} u_{i k} \otimes u_{k j}
$$

is a $*$-homomorphism. A CMQG $(A, u)$ is a quantum subgroup of $(B, v)$, if there is a surjective *-homomorphism from $B$ to $A$ mapping $v_{i j}$ to $u_{i j}$.

Of course, in the definition of a CMQG $(A, u)$, we should think of $A$ as the functions on the "quantum space" $\mathbb{G}_{A}$ (and $(B, v)$ as associated to $\mathbb{G}_{B}$ ). Gelfand duality, which is contravariant, therefore explains why the "inclusion $\mathbb{G}_{A} \hookrightarrow \mathbb{G}_{B}$ " is represented by a surjective morphism $B \rightarrow A$.

In this paper, we use the notations $\mathbb{N}_{0}=\{0,1,2, \ldots\}$ and $\mathbb{N}=\{1,2, \ldots\}$ (as opposed to [19], where $\mathbb{N}=\{0,1, \ldots\})$.

\subsection{Categories of partitions and easy quantum groups}

Colored partitions are key tools for the introduction of unitary easy quantum groups, as done in [37]. They generalize the orthogonal easy quantum groups of Banica and Speicher [5]. Let $k, l \in \mathbb{N}_{0}$ and consider a finite ordered set with $k+l$ elements each being colored either in white or in black. A partition is a decomposition of this set into disjoint subsets, the blocks. Let 
$P^{\circ \bullet}(k, l)$ denote the set of all such partitions and put $P^{\circ \bullet}:=\bigcup_{k, l \in \mathbb{N}_{0}} P^{\circ}(k, l)$. We usually use a pictorial representation of a partition involving lines representing the block structure, and we assume $k$ of these points to be placed on an upper row and $l$ on a lower row, see [36]. If these lines may be drawn in a way such that they do not cross, we call the partition noncrossing. Let $N C^{\circ \bullet}$ be the set of all noncrossing partitions. Some examples of partitions are the singleton partitions $\uparrow \in P^{\circ} \bullet(0,1)$ consisting of a single white lower point or $\uparrow \in P^{\circ \bullet}(0,1)$, the pair partitions $\square \bullet$ and $\square$ in $P(0,2)$ consisting of two lower points of different colors which are in the same block, the identity partitions $\stackrel{0}{0}, \in P(1,1)$ consisting of one upper and one lower point both of the same color and both in the same block, or the partitions $b_{s} \in P^{\circ \bullet}(0, s)$ consisting of $s$ lower white points in a single block.

We have the following operations on the set $P^{\circ}$ of partitions. The tensor product of $p \in$ $P^{\circ \bullet}(k, l)$ and $q \in P^{\circ \bullet}\left(k^{\prime}, l^{\prime}\right)$ is $p \otimes q \in P^{\circ \bullet}\left(k+k^{\prime}, l+l^{\prime}\right)$ obtained by placing $p$ and $q$ side by side. The composition of $p \in P^{\circ \bullet}(k, l)$ and $q \in P^{\circ \bullet}(l, m)$ is $q p \in P^{\circ \bullet}(k, m)$ obtained by placing $p$ above $q$. We may only perform it when the color pattern of the lower points of $p$ matches the upper color pattern of $q$. The involution of $p \in P^{\circ \bullet}(k, l)$ is $p^{*} \in P^{\bullet \bullet}(l, k)$ obtained by reflecting $p$ at the horizontal axis. The rotation of the left upper point of $p \in P^{\circ}(k, l)$ to the lower row is a partition in $P^{\circ \bullet}(k-1, l+1)$. When rotating a point, its color is inverted but its membership to a block remains untouched. Likewise we have a rotation of the left lower or right upper/lower points. These operations (tensor product, composition, involution and rotation) are called the category operations.

A collection $\mathcal{C}$ of subsets $\mathcal{C}(k, l) \subseteq P^{\circ}(k, l)$ (for every $\left.k, l \in \mathbb{N}_{0}\right)$ is a category of partitions if it is invariant under the category operations and if the identity partitions $0, \bullet \in P^{\circ}(1,1)$ and the pair partitions $\sqcap, \curvearrowleft_{0} \in P^{\circ}(0,2)$ are in $\mathcal{C}$. Note that rotation may be deduced from the other category operations. We write $\mathcal{C}=\left\langle p_{1}, \ldots, p_{m}\right\rangle$, if $\mathcal{C}$ is the smallest category of partitions containing the partitions $p_{1}, \ldots, p_{m}$. We say that $\mathcal{C}$ is generated by $p_{1}, \ldots, p_{m}$.

Let $n \in \mathbb{N}$. Given $p \in P^{\circ}(k, l)$ and two multi-indices $(i(1), \ldots, i(k)),(j(1), \ldots, j(l))$ with entries in $\{1, \ldots, n\}$, we can label the diagram of $p$ with these numbers (the upper and the lower row both are labelled from left to right, respectively) and we put

$$
\delta_{p}(i, j)= \begin{cases}1, & \text { if each block of } p \text { connects only equal indices } \\ 0, & \text { otherwise }\end{cases}
$$

We fix a basis $e_{1}, \ldots, e_{n}$ of $\mathbb{C}^{n}$ and define a map $T_{p}:\left(\mathbb{C}^{n}\right)^{\otimes k} \rightarrow\left(\mathbb{C}^{n}\right)^{\otimes l}$ associated to $p$ by

$$
T_{p}\left(e_{i(1)} \otimes \cdots \otimes e_{i(k)}\right)=\sum_{1 \leq j(1), \ldots, j(l) \leq n} \delta_{p}(i, j) \cdot e_{j(1)} \otimes \cdots \otimes e_{j(l)} .
$$

We use the convention that $\left(\mathbb{C}^{n}\right)^{\otimes 0}=\mathbb{C}$, i.e., for a partition $p \in P^{\circ}(0, l)$ with no upper points, $T_{p}(1)$ is actually a vector in $\left(\mathbb{C}^{n}\right)^{\otimes l}$. Note that the colors of the points of $p$ do not play a role in the definition of $T_{p}$. The operations on the partitions match nicely with canonical operations of the linear maps $T_{p}$, namely we have $T_{p \otimes q}=T_{p} \otimes T_{q}, T_{q p}=n^{-b(p, q)} T_{q} T_{p}$ and $T_{p^{*}}=\left(T_{p}\right)^{*}$. Here, $b(p, q)$ denotes the number of removed blocks when composing $p$ and $q$. The maps $T_{p}$ can be normalized in such a way that they become partial isometries, see [18].

From a category $\mathcal{C}$ and the realisation $p \mapsto T_{p}$, we may construct a concrete monoidal $W^{*}$ category with a distinguished object $u$, and we thus may assign a CMQG $(A, u)$ to it. We call it the easy quantum group associated to $\mathcal{C}$. If $\mathcal{C} \subset N C^{\circ}$, then it is called a free easy quantum group. Given a color string $r \in\{\circ, \bullet\}^{k}$, we may define $u^{r}$ as the tensor product of copies $u$ and $\bar{u}$. Here $\circ$ corresponds to $u$ while $\bullet$ corresponds to $\bar{u}$. We then may say: a CMQG $S_{n} \subset \mathbb{G} \subset U_{n}^{+}$is 
easy, if there is a category of partitions $\mathcal{C}$ such that the intertwiner spaces of $\mathbb{G}$ are of the form

$$
\left\{T \mid T u^{r}=u^{s} T\right\}=\operatorname{span}\left\{T_{p} \mid p \in \mathcal{C}(k, l) \text { with color strings } r \text { (upper) and } s \text { (lower) }\right\} .
$$

Easy quantum groups are a class of CMQGs with a quite intrinsic combinatorial structure. They are completely determined by their associated category of partitions and in many cases certain quantum algebraic properties of an easy quantum group may be traced back to certain combinatorial properties of its category of partitions. A simple criterion for verifying that a given CMQG is easy is contained in the following lemma.

Let $n \in \mathbb{N}$ and let $A$ be a $C^{*}$-algebra generated by $n^{2}$ elements $u_{i j}, 1 \leq i, j \leq n$. Let $p \in P^{\circ} \bullet(k, l)$ be a partition with upper color string $r \in\{\circ, \bullet\}^{k}$ and lower color string $s \in\{\circ, \bullet\}^{l}$. We say that the generators $u_{i j}$ fulfill the relations $R(p)$, if for all $\beta(1), \ldots, \beta(l) \in\{1, \ldots, n\}$ and for all $i(1), \ldots, i(k) \in\{1, \ldots, n\}$, we have

$$
\sum_{\alpha(1), \ldots, \alpha(k)=1}^{n} \delta_{p}(\alpha, \beta) u_{\alpha(1) i(1)}^{r_{1}} \cdots u_{\alpha(k) i(k)}^{r_{k}}=\sum_{\gamma(1), \ldots, \gamma(l)=1}^{n} \delta_{p}(i, \gamma) u_{\beta(1) \gamma(1)}^{s_{1}} \cdots u_{\beta(l) \gamma(l)}^{s_{l}} .
$$

The left-hand side of the equation is $\delta_{p}(\varnothing, \beta)$ if $k=0$ and analogously $\delta_{p}(i, \varnothing)$ for the right-hand side if $l=0$. Furthermore, $u_{i j}^{\circ}:=u_{i j}$ and $u_{i j}^{\bullet}:=u_{i j}^{*}$.

Lemma 2.2 ([37, Corollary 3.12]). Let $p_{1}, \ldots, p_{m} \in P^{\circ}$ be partitions and let $A$ be the universal $C^{*}$-algebra generated by elements $u_{i j}, 1 \leq i, j \leq n$ such that $u=\left(u_{i j}\right)$ and $\bar{u}=\left(u_{i j}^{*}\right)$ are unitary (i.e., $\left.\sum_{k} u_{i k}^{*} u_{j k}=\sum_{k} u_{k i}^{*} u_{k j}=\sum_{k} u_{i k} u_{j k}^{*}=\sum_{k} u_{k i} u_{k j}^{*}=\delta_{i j}\right)$ and $u_{i j}$ satisfy relations $R\left(p_{l}\right)$ for $l=1, \ldots, m$. Then $A$ is an easy quantum group with associated category $\mathcal{C}=\left\langle p_{1}, \ldots, p_{m}\right\rangle$.

Proof. The proof relies on the fact that the relations $R(p)$ are fulfilled if and only if $T_{p}$ intertwines $u^{r}$ and $u^{s}$. See [37, Lemma 3.9, Corollary 3.12] for details.

Note that for $p=$ ? the relation $R(p)$ effects that all $u_{i j}$ are selfadjoint. On the combinatorial level this amounts to having partitions whose points have no colors. In this case, we recover the orthogonal easy quantum groups of Banica and Speicher [5].

\section{Example 2.3.}

(a) The free orthogonal quantum group $O_{n}^{+}$is given by the universal unital $C^{*}$-algebra $C\left(O_{n}^{+}\right)$ generated by selfadjoint elements $u_{i j}, 1 \leq i, j \leq n$ such that $u=\left(u_{i j}\right)$ is an orthogonal matrix. It is easy with category $\mathcal{C}=\langle\mathfrak{\bullet}\rangle=N C_{2}$, the set of all (noncolored) noncrossing pair partitions (each block consists of exactly two elements). We omit to write down the

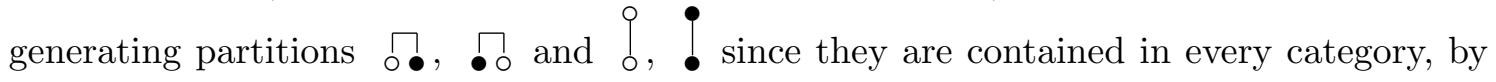
definition.

(b) The free unitary quantum group $U_{n}^{+}$is given by the universal unital $C^{*}$-algebra $C\left(U_{n}^{+}\right)$ generated by elements $u_{i j}, 1 \leq i, j \leq n$ such that $u=\left(u_{i j}\right)$ and $\bar{u}=\left(u_{i j}^{*}\right)$ are unitary matrices. It is an easy quantum group with $\mathcal{C}=\langle\varnothing\rangle$.

(c) The quantum permutation group $S_{n}^{+}$is given by the universal unital $C^{*}$-algebra $C\left(S_{n}^{+}\right)$ generated by projections $u_{i j}, 1 \leq i, j \leq n$ such that $\sum_{k} u_{i k}=\sum_{k} u_{k j}=1$. It is easy with

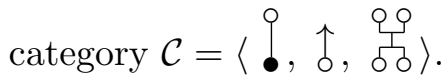

The quantum groups in (a), (b) and (c) were introduced by Wang [39, 40]. 
(d) For $s \in \mathbb{N}$, the quantum reflection group $H_{n}^{s+}$ is given by the universal unital $C^{*}$-algebra $C\left(H_{n}^{s+}\right)$ generated by elements $u_{i j}, 1 \leq i, j \leq n$ such that $u=\left(u_{i j}\right)$ and $\bar{u}=\left(u_{i j}^{*}\right)$ are unitaries, all $u_{i j}$ are partial isometries and we have $u_{i j}^{s}=u_{i j} u_{i j}^{*}=u_{i j}^{*} u_{i j}$. We have $H_{n}^{1+}=S_{n}^{+}$and $H_{n}^{2+}=H_{n}^{+}$, the latter one being Banica, Bichon and Collins's hyperoctahedral quantum group [4]. The quantum reflection groups $H_{n}^{s+}$ were studied by Banica,

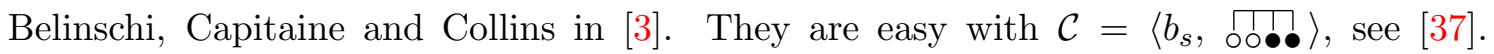
Both $H_{n}^{+}$and $H_{n}^{s+}$ can be traced back to Bichon's work on free wreath product [9].

\subsection{Cuntz algebra}

The Cuntz algebra $\mathcal{O}_{n}$ is the universal unital $C^{*}$-algebra generated by isometries $S_{1}, \ldots, S_{n}$ such that $\sum_{i} S_{i} S_{i}^{*}=1$. It has been defined and studied by Cuntz in [11]. He first proved that $\mathcal{O}_{n}$ is a crossed product by an endomorphism and then introduced a criterion for (what we call today) purely infinite and simple algebras. He used it to show that all $\mathcal{O}_{n}$ are simple.

By an action of a CQG on a $C^{*}$-algebra $A$ we mean a faithful unital *-homomorphism $\alpha: A \rightarrow A \otimes C(\mathbb{G})$ such that $(\alpha \otimes \operatorname{Id}) \circ \alpha=(\operatorname{Id} \otimes \Delta) \circ \alpha$ holds and $(1 \otimes C(\mathbb{G})) \alpha(A)$ is linearly dense in $A \otimes C(\mathbb{G})$. In our case, we have the following action on the Cuntz algebra, as observed by Cuntz [12] (see also [25]).

Proposition 2.4. Let $\mathbb{G}$ be a $C M Q G$ such that $\mathbb{G} \subset U_{n}^{+}$(i.e., the matrices $u$ and $\bar{u}$ are unitaries). It acts on $\mathcal{O}_{n}$ by

$$
\alpha\left(S_{i}\right)=\sum_{j=1}^{n} S_{j} \otimes u_{j i} .
$$

Proof. For the convenience of the reader, we give the proof since it is a short argument. By the universal property of $\mathcal{O}_{n}$, the unital $*$-homomorphism $\alpha$ exists, and it is faithful since $\mathcal{O}_{n}$ is simple. It is straightforward to check that $(\alpha \otimes \operatorname{Id}) \circ \alpha=(\operatorname{Id} \otimes \Delta) \circ \alpha$ is satisfied, and the computation (using orthogonality of $u$ )

$$
\sum_{k}\left(1 \otimes u_{i k}^{*}\right)\left(\sum_{l} S_{l} \otimes u_{l k}\right)=S_{i} \otimes 1
$$

shows that the linear span of $(1 \otimes C(\mathbb{G})) \alpha(A)$ equals $A \otimes C(\mathbb{G})$.

It is a well-known fact that we may find copies of matrix algebras inside the Cuntz algebra. Indeed, the monomials $S_{j(1)} \cdots S_{j(k)} S_{i(k)}^{*} \cdots S_{i(1)}^{*}$ satisfy the relations of matrix units, thus we have

$$
\operatorname{span}\left\{S_{j(1)} \cdots S_{j(k)} S_{i(k)}^{*} \cdots S_{i(1)}^{*} \mid 1 \leq i(t), j(t) \leq d\right\} \cong B\left(\left(\mathbb{C}^{n}\right)^{\otimes k}\right) .
$$

Thus, $S_{j(1)} \cdots S_{j(k)} S_{i(k)}^{*} \cdots S_{i(1)}^{*}$ corresponds exactly to the rank one operator mapping $e_{i(1)} \otimes$ $\cdots \otimes e_{i(k)}$ to $e_{j(1)} \otimes \cdots \otimes e_{j(k)}$. More generally, we may identify linear maps

$$
\begin{aligned}
T:\left(\mathbb{C}^{n}\right)^{\otimes k} & \rightarrow\left(\mathbb{C}^{n}\right)^{\otimes l}, \\
e_{i(1)} \otimes \cdots \otimes e_{i(k)} & \mapsto \sum_{j(1), \ldots, j(l)} a(i(1), \ldots, i(k), j(1), \ldots, j(l)) e_{j(1)} \otimes \cdots \otimes e_{j(l)}
\end{aligned}
$$

(where $a(i(1), \ldots, i(k), j(1), \ldots, j(l)) \in \mathbb{C})$ with elements

$$
\sum_{i(1), \ldots, i(k), j(1), \ldots, j(l)=1} a(i(1), \ldots, i(k), j(1), \ldots, j(l)) S_{j(1)} \cdots S_{j(l)} S_{i(k)}^{*} \cdots S_{i(1)}^{*}
$$


in $\mathcal{O}_{n}$. Hence, we may view the maps $T_{p}:\left(\mathbb{C}^{n}\right)^{\otimes k} \rightarrow\left(\mathbb{C}^{n}\right)^{\otimes l}$ indexed by partitions $p \in P(k, l)$ as elements in $\mathcal{O}_{n}$ via

$$
T_{p} \leftrightarrow \sum_{i(1), \ldots, i(k), j(1), \ldots, j(l)=1}^{n} \delta_{p}(i, j) S_{j(1)} \cdots S_{j(l)} S_{i(k)}^{*} \cdots S_{i(1)}^{*}
$$

\section{Actions of easy quantum groups on the Cuntz algebra}

\subsection{The fixed point algebra}

As explained in Section 2.3, any given CQMG acts naturally on a Cuntz algebra $\mathcal{O}_{n}$ for a suitable choice of $n$. Our aim is to understand the fixed point algebra in the case of easy quantum groups.

Definition 3.1. Let $\mathbb{G}$ be a CMQG with fundamental representation $u=\left(u_{i j}\right)$ and let $\alpha$ be the action as in Proposition 2.4. The fixed point algebra $\mathcal{O}^{\alpha}$ is defined as

$$
\mathcal{O}^{\alpha}:=\left\{x \in \mathcal{O}_{n} \mid \alpha(x)=x \otimes 1\right\} .
$$

In the case of easy quantum groups, we may read the fixed point algebras directly from the categories of partitions. The following statement appeared in [27, Proposition 3.4], see also [19, Lemma 2.5].

Proposition 3.2. Given $\mathcal{C}$, let $\mathbb{G}$ be the associated easy $Q G$. The intersections of the fixed point algebra $\mathcal{O}^{\alpha}$ with the copies of $B\left(\left(\mathbb{C}^{n}\right)^{\otimes k},\left(\mathbb{C}^{n}\right)^{\otimes l}\right)$ in $\mathcal{O}_{n}$ (as described in Section 2.3) are given by

$$
\mathcal{O}^{\alpha} \cap B\left(\left(\mathbb{C}^{n}\right)^{\otimes k},\left(\mathbb{C}^{n}\right)^{\otimes l}\right)=\operatorname{span}\left\{T_{p} \in \mathcal{O}_{n} \mid p \in \mathcal{C}(k, l) \text { all points are white }\right\} .
$$

Proof. The general proof may be found in [27, Proposition 3.4], but we give a direct argument here. Let $p \in \mathcal{C}(k, l)$ be a partition with only white points. Hence, in $C(\mathbb{G})$ the relations $R(p)$ hold. Moreover, we use the fact that $u$ is unitary. Now

$$
\begin{aligned}
\alpha\left(T_{p}\right)= & \sum_{i, i^{\prime}, j, j^{\prime}} \delta_{p}(i, j) S_{j^{\prime}(1)} \cdots S_{j^{\prime}(l)} S_{i^{\prime}(k)}^{*} \cdots S_{i^{\prime}(1)}^{*} \otimes u_{j^{\prime}(1) j(1)} \cdots u_{j^{\prime}(l) j(l)} u_{i^{\prime}(k) i(k)}^{*} \cdots u_{i^{\prime}(1) i(1)}^{*} \\
= & \sum_{i^{\prime}, j^{\prime}} S_{j^{\prime}(1)} \cdots S_{j^{\prime}(l)} S_{i^{\prime}(k)}^{*} \cdots S_{i^{\prime}(1)}^{*} \\
& \otimes\left(\sum_{i}\left(\sum_{j} \delta_{p}(i, j) u_{j^{\prime}(1) j(1)} \cdots u_{j^{\prime}(l) j(l)}\right) u_{i^{\prime}(k) i(k)}^{*} \cdots u_{i^{\prime}(1) i(1)}^{*}\right) \\
= & \sum_{i^{\prime}, j^{\prime}} S_{j^{\prime}(1)} \cdots S_{j^{\prime}(l)} S_{i^{\prime}(k)}^{*} \cdots S_{i^{\prime}(1)}^{*} \\
& \otimes\left(\sum_{i}\left(\sum_{s} \delta_{p}\left(s, j^{\prime}\right) u_{s(1) i(1)} \cdots u_{s(k) i(k)}\right) u_{i^{\prime}(k) i(k)}^{*} \cdots u_{i^{\prime}(1) i(1)}^{*}\right) \\
= & \sum_{i^{\prime}, j^{\prime}} S_{j^{\prime}(1)} \cdots S_{j^{\prime}(l)} S_{i^{\prime}(k)}^{*} \cdots S_{i^{\prime}(1)}^{*} \\
& \otimes\left(\sum_{s} \delta_{p}\left(s, j^{\prime}\right)\left(\sum_{i} u_{s(1) i(1)} \cdots u_{s(k) i(k)} u_{i^{\prime}(k) i(k)}^{*} \cdots u_{i^{\prime}(1) i(1)}^{*}\right)\right) \\
= & \sum_{i^{\prime}, j^{\prime}} S_{j^{\prime}(1)} \cdots S_{j^{\prime}(l)} S_{i^{\prime}(k)}^{*} \cdots S_{i^{\prime}(1)}^{*} \otimes\left(\sum_{s} \delta_{p}\left(s, j^{\prime}\right) \delta_{s i^{\prime}}\right) \\
= & \sum_{j^{\prime}} \delta_{p}\left(i^{\prime}, j^{\prime}\right) S_{j^{\prime}(1)} \cdots S_{j^{\prime}(l)} S_{i^{\prime}(k)}^{*} \cdots S_{i^{\prime}(1)}^{*} \otimes 1=T_{p} \otimes 1 .
\end{aligned}
$$


Conversely, let $T \in \mathcal{O}^{\alpha} \cap B\left(\left(\mathbb{C}^{n}\right)^{\otimes k},\left(\mathbb{C}^{n}\right)^{\otimes l}\right)$. Reversing the above computation, we see that $T$ intertwines $u^{\otimes k}$ and $u^{\otimes l}$. But $\operatorname{Hom}\left(u^{\otimes k}, u^{\otimes l}\right)$ consists exactly of the linear span of all $T_{p} \in \mathcal{O}_{n}$ such that $p \in \mathcal{C}(k, l)$ has only white points.

Proposition 3.3. The algebraic result of Proposition 3.2 extends into a topological one, namely,

$$
\mathcal{O}^{\alpha}=\overline{\operatorname{span}\left\{T_{p} \in \mathcal{O}_{n} \mid p \in \mathcal{C}(k, l) \text { all points are white, } \forall k, l \in \mathbb{N}_{0}\right\}} \text {. }
$$

Proof. Lemma 2.7 of [19, p. 1017] (see also [25, Lemma 6]) ensures that "algebraic elements" (i.e., those obtained by (finite) polynomial combination of $S_{j}$ ) of $\mathcal{O}^{\alpha}$ are dense in $\mathcal{O}^{\alpha}$. Proposition 3.2 above ensures that any such algebraic element is in the linear span of $T_{p}$, so the conclusion follows.

\subsection{Obtaining Kirchberg algebras}

In [19], the first author isolated two conditions which turn $\mathcal{O}^{\alpha}$ into a Kirchberg algebra. This class of algebras plays a central role in Kirchberg-Phillips classification theory, which proves that Kirchberg algebras are completely classified by their $K$-groups - see [23, 24] for the original papers, [35] for an overview of classification for nuclear simple $C^{*}$-algebras and [38] for the latest developments in this area.

We denote by $\mathcal{T}_{u}$ the set of (classes of) irreducible representations appearing in the iterated tensor products $u^{\otimes l}$ for $l \in \mathbb{N}_{0}$.

(C1) For any $v \in \mathcal{T}_{u}$, we can find $v^{\prime} \in \mathcal{T}_{u}$ such that the representation $v \otimes v^{\prime}$ possesses a nonzero invariant vector.

(C2) There are integers $N, k_{0} \in \mathbb{N}_{0}$ such that $u^{\otimes N}$ is contained in $u^{\otimes\left(N+k_{0}\right)}$ and for all integers $t, l$ with $0<t<k_{0}, \operatorname{Hom}\left(u^{\otimes l}, u^{\otimes(l+t)}\right)=0$.

Condition (C1) is actually satisfied for all finite-dimensional semisimple Hopf algebras over $\mathbb{C}$, see [19, Remark 7.5] and [22, Theorem of Section 4.2]. The following result has been proven by the first author, see [19, Lemma 2.10, Corollary 4.7, Lemma 6.4]. It holds true in a much more general setting but we restrict it to CMQG.

Proposition 3.4. Let $\mathbb{G}$ be a $C M Q G$ and let $\alpha$ be its action on $\mathcal{O}_{n}$ as described in Proposition 2.4. If the conditions $(\mathrm{C} 1)$ and $(\mathrm{C} 2)$ are satisfied, then the fixed point algebra $\mathcal{O}^{\alpha}$ is a Kirchberg algebra, i.e., it is purely infinite, simple, separable, unital and nuclear (satisfying the UCT).

We are now going to study which easy quantum groups satisfy conditions $(\mathrm{C} 1)$ and $(\mathrm{C} 2)$. In order to do so, let us rephrase these conditions in the language of partitions. The representation theory of easy quantum groups - i.e., the set $\mathcal{T}_{u}$ - is completely understood and can be given in terms of partitions, see [18]. We review some facts from [18].

We say that a partition $p \in P^{\circ \bullet}(k, k)$ is projective, if $p=p^{*}=p^{2}$. It follows that $T_{p}$ is a projection up to normalization. Moreover, the upper points of $p$ are colored exactly like the lower points of $p$. If $q \in P^{\circ \bullet}(k, k)$ is another projective partition, we write $q \prec p$ if $p q=q p=q$ and $p \neq q$. In this case, $T_{q}$ is a subprojection of $T_{p}$. Given a category $\mathcal{C}$ of partitions, we write $\operatorname{Proj}_{\mathcal{C}}(k)$ for the set of all projective partitions in $\mathcal{C}(k, k)$. For $p \in \operatorname{Proj}_{\mathcal{C}}(k)$ with upper (and equivalently lower) color string $s$, we put

$$
R_{p}:=\bigvee_{q \in \operatorname{Proj}_{\mathcal{C}}(k), q \prec p} T_{q}, \quad \text { and } \quad P_{p}:=T_{p}-R_{p} \in \operatorname{Hom}\left(u^{s}, u^{s}\right) .
$$

We denote by $u_{p}$ the subrepresentation $\left(\operatorname{Id} \otimes P_{p}\right)\left(u^{s}\right)$ of $u^{s}$. Two such subrepresentations $u_{p}$ and $u_{q}$ are unitarily equivalent if and only if there is a partition $r \in \mathcal{C}$ such that $p=r^{*} r$ and $q=r r^{*}$. 
Proposition 3.5 ([18, Theorem 5.5]). If $\mathcal{C} \subset N C^{\circ}$, then $u_{p}$ is irreducible. Furthermore, any irreducible representation of the associated $\mathbb{G}$ is unitarily equivalent to some $u_{p}$ for some $p \in \operatorname{Proj}_{\mathcal{C}}$, thereby inducing a one-to-one correspondence.

Since only tensor powers of $u$ play a role for conditions (C1) and (C2), let us denote by $\operatorname{Proj}_{\mathcal{C}}^{\text {white }}$ and $\mathcal{C}^{\text {white }}$ the restrictions of $\operatorname{Proj}_{\mathcal{C}}$ and $\mathcal{C}$ to those partitions consisting only of white points.

Theorem 3.6. Let $\mathbb{G}$ be a free easy quantum group, namely the associated category of partitions $\mathcal{C}$ contains only noncrossing partitions. The following two conditions imply the conditions $(\mathrm{C} 1)$ and $(\mathrm{C} 2)$.

$\left(\mathrm{C}_{P} 1\right)$ For any projective partition $p \in \operatorname{Proj}_{\mathcal{C}}^{\text {white }}(a)$, we can find a projective partition $q \in$ $\operatorname{Proj}_{\mathcal{C}}^{\text {white }}(b)$ and a partition $r \in \mathcal{C}^{\text {white }}(0, a+b)$ with no upper points such that $\left(P_{p} \otimes P_{q}\right) T_{r} \neq 0$.

$\left(\mathrm{C}_{P} 2\right)$ There are integers $k_{0}, N \in \mathbb{N}_{0}$ and a partition $r \in \mathcal{C}^{\text {white }}\left(N+k_{0}, N\right)$ such that $r r^{*}=0^{\otimes N}$. Moreover, for all $t \in \mathbb{N}_{0}$ with $0<t<k_{0}$ and for all $l \in \mathbb{N}_{0}$ we have $\mathcal{C}^{\text {white }}(l, l+t)=\varnothing$.

Proof. Due to Proposition 3.5, the set $\mathcal{T}_{u}$ is in bijection with equivalence classes of projective partitions in $\mathcal{C}$. Let us first prove that $\left(\mathrm{C}_{P} 1\right)$ implies $(\mathrm{C} 1)$. Let $p \in \operatorname{Proj}_{\mathcal{C}}$ be a projective partition with only white points and let $u_{p} \in \mathcal{T}_{u}$ be the associated irreducible representation. Let $q \in \operatorname{Proj}_{\mathcal{C}}$ and $r \in \mathcal{C}(0, a+b)$ be partitions according to $\left(\mathrm{C}_{P} 1\right)$. Then $T_{r}(1)$ is a vector in $\left(\mathbb{C}^{n}\right)^{\otimes a+b}$ as described in Section 2.2 and hence $\left(P_{p} \otimes P_{q}\right) T_{r}(1)$ is a non-zero vector. Now, since $P_{p}, P_{q}$ and $T_{r}$ are intertwiners for tensor powers of $u$, we have:

$$
\begin{aligned}
\left(u_{p} \otimes u_{q}\right)\left(P_{p} \otimes P_{q}\right) T_{r} & =\left(\operatorname{Id} \otimes\left(P_{p} \otimes P_{q}\right)\right)\left(u^{\otimes a+b}\right)\left(P_{p} \otimes P_{q}\right) T_{r} \\
& =\left(\operatorname{Id} \otimes\left(P_{p} \otimes P_{q}\right)\right)\left(u^{\otimes a+b}\right) T_{r}=\left(P_{p} \otimes P_{q}\right) T_{r} .
\end{aligned}
$$

This proves (C1). As for deducing (C2) from $\left(\mathrm{C}_{P} 2\right)$, observe that $u^{\otimes N} T_{r}=T_{r} u^{\otimes N+k_{0}}$ implies $u^{\otimes N} T_{r} T_{r}^{*}=T_{r} u^{\otimes N+k_{0}} T_{r}^{*}$. Since $T_{r} T_{r}^{*}=1$ up to normalization, this proves that $u^{\otimes N}$ is a subrepresentation of $u^{\otimes N+k_{0}}$. Moreover, $\operatorname{Hom}\left(u^{\otimes l}, u^{\otimes(l+t)}\right)$ is spanned by all $T_{p}$ with $p \in \mathcal{C}^{\text {white }}(l, l+t)$. We have proved $(\mathrm{C} 2)$.

The advantage of dealing with free easy quantum groups is that they are all known: the class of categories $\mathcal{C} \subset N C^{\circ}$ - and hence the class of free easy quantum groups - is completely classified. The complete list may be found in [36, Section 7].

Throughout the classification process, a parameter $k(\mathcal{C}) \in \mathbb{N}_{0}$ is assigned to any category of partitions $\mathcal{C}$, see [36, Definition 2.5]. It is given by the following. For a partition $p \in P^{\circ} \bullet$ denote by $c_{\circ}(p)$ the sum of the number of white points on the lower line of $p$ and the number of black points on its upper line. Likewise put $c_{\bullet}(p)$ to be the number of lower black points plus the number of upper white points. Let $c(p):=c_{\circ}(p)-c_{\bullet}(p)$. The number $c(p)$ is designed in such a way that it yields the difference of the number of white and black points, if $p$ has no upper points; moreover $c(p)$ is invariant under rotation. Now, let $k(\mathcal{C})$ be the minimum of all numbers $c(p)>0$ for $p \in \mathcal{C}$ if such a number exists, and $k(\mathcal{C}):=0$ otherwise. One can show that for every partition $p \in \mathcal{C}$ the number $c(p)$ is a multiple (from the integers) of $k(\mathcal{C})$. One of the main results in [37] is then to detect the cyclic group of order $k(\mathcal{C})$ as a building block in the easy quantum group associated to $\mathcal{C}$. The parameter $k(\mathcal{C})$ also appears in connection with conditions $\left(\mathrm{C}_{P} 1\right)$ and $\left(\mathrm{C}_{P} 2\right)$.

Theorem 3.7. Let $\mathbb{G}$ be an easy quantum group with $\mathcal{C} \subset N C^{\circ}$.

(a) If $k(\mathcal{C})=0$, then neither condition $\left(\mathrm{C}_{P} 1\right)$ nor $\left(\mathrm{C}_{P} 2\right)$ are satisfied. 
(b) If $k(\mathcal{C}) \neq 0$ and $\square \otimes \bullet \otimes \in \mathcal{C}$, then condition $\left(\mathrm{C}_{P} 1\right)$ holds.

(c) If $k(\mathcal{C}) \neq 0$ and $\prod_{\bullet \bullet \bullet} \in \mathcal{C}$, then condition $\left(\mathrm{C}_{P} 1\right)$ holds.

(d) If $k(\mathcal{C}) \neq 0$, then condition $\left(\mathrm{C}_{P} 2\right)$ holds.

Proof. (a) Observe that if a partition $p$ is in $\mathcal{C}^{\text {white }}(l, l+t)$, then $c(p)=t$. Thus, if $k(\mathcal{C})=0$, all sets $\mathcal{C}^{\text {white }}(l, l+t)$ are empty for all $l \in \mathbb{N}_{0}$ and all $t \neq 0$, using [36, Proposition 2.7]. Therefore neither condition $\left(\mathrm{C}_{P} 1\right)$ nor condition $\left(\mathrm{C}_{P} 2\right)$ hold.

(b) Let $p \in \operatorname{Proj}_{\mathcal{C}}^{\text {white }}(a)$ be a projective partition. We basically want to show that the contragredient representation of $u_{p}$ does the job for choosing $q$, but the colorization of the points turns this into a nontrivial problem (see also the next Examples 3.8 and the remarks in $\left[19\right.$, p. 1019] on condition $(\mathrm{C} 1)$ ). Let $q_{1}$ be the partition obtained by reflecting $p$ about the vertical axis (without inverting the colors). It is in $\mathcal{C}$, since the verticolor reflected partition $\tilde{p}$ is in $\mathcal{C}$ [36, Lemma 1.1(a)], and $p \otimes p \otimes \tilde{p}$ is in $\mathcal{C}$; using color permutation [36, Lemmas 1.3(a) and 1.1(b)], we infer $q_{1} \in \mathcal{C}$. Now, let $b \in \mathbb{N}_{0}$ be such that $2(a+b)$ is a multiple of $k(\mathcal{C})$. Let $q_{0}=\sqcap \square^{\text {nest }(b)} \otimes \bigcirc^{\text {nest }(b)}$ be the partition obtained by nesting $b$ copies of the pair partition $\square \circ$ into each other, both on the upper and the lower line respectively, see [37, Lemma 2.4]. It is in $\mathcal{C}$ since we may apply [36, Lemmas $1.3(\mathrm{a})$ and $1.1(\mathrm{~b})]$ on the following partition which is in $\mathcal{C}$ :

$$
\left.\square^{\operatorname{nest}(b)} \otimes \stackrel{\circ}{\bullet^{\operatorname{nest}(b)} \otimes(\sqcap \circ \otimes \bullet \bullet}\right)^{\otimes b} .
$$

We then put $q:=q_{0} \otimes q_{1} \in \operatorname{Proj}_{\mathcal{C}}^{\text {white }}(a+2 b)$ and $r:=\bigcap_{\circ}^{\text {nest }(a+b)}$. We have $r \in \mathcal{C}^{\text {white }}(0,2(a+b))$, see for instance Step 3 in the proof of [37, Theorem 4.13].

If now $s \in \operatorname{Proj}_{\mathcal{C}}^{\text {white }}(a)$ is a projective partition with $s \prec p$, then $(s \otimes q) r \neq(p \otimes q) r$ by [18, Lemma 2.23]. Hence $T_{(s \otimes q) r}$ is linearly independent from $T_{(p \otimes q) r}$ by [18, Lemma 4.16]. Likewise $T_{(p \otimes t) r}$ is linearly independent from $T_{(p \otimes q) r}$ for $t \prec q, t \in \operatorname{Proj}_{\mathcal{C}}^{\text {white }}(a+2 b)$. This proves that $\left(T_{p} \otimes T_{q}\right) T_{r}$ is linearly independent from $\left(R_{p} \otimes T_{q}\right) T_{r},\left(T_{p} \otimes R_{q}\right) T_{r}$ and $\left(R_{p} \otimes R_{q}\right) T_{r}$. Thus, $\left(P_{p} \otimes P_{q}\right) T_{r} \neq 0$.

(c) Let $p \in \operatorname{Proj}_{\mathcal{C}}^{\text {white }}(a)$ be a projective partition. Using the through-block decomposition of $p$, we may bring it into the following form (see Proposition 2.9 and the remarks after Proposition 2.12 in [18]):

$$
p=p_{u}^{*} p_{u}, \quad p_{u}=s_{0} \otimes t_{1} \otimes s_{1} \otimes \cdots \otimes t_{l} \otimes s_{l} .
$$

Here, $s_{i}$ are partitions with no upper points while each $t_{i}$ has exactly one upper point. Let $\alpha_{i}$ be the sum of the number of points of $s_{i}$ and $t_{i}$, with $\alpha_{1}$ being the sum of the number of points of $s_{0}, t_{1}$ and $s_{1}$. Let $\beta_{i}$ be numbers such that $\alpha_{i}+\beta_{i}$ is a multiple of $k(\mathcal{C})$, for all $i$. Let $q$ be the partition

$$
q:=q_{1} \otimes \cdots \otimes q_{l}
$$

where each $q_{i}$ is the partition consisting of a single block on $\beta_{i}$ upper white points and $\beta_{i}$ lower white points. Since $\prod_{0 \circ \bullet} \in \mathcal{C}$, we have $q \in \mathcal{C}$, applying [36, Lemma $\left.1.3(\mathrm{c})\right]$ on ${ }^{0}$. Let $r \in \mathcal{C}^{\text {white }}\left(0, \sum_{i}\left(\alpha_{i}+\beta_{i}\right)\right)$ be the partition obtained from nesting $l$ blocks $b_{i}$ into each other, each of size $\alpha_{i}+\beta_{i}$, such that block $b_{i+1}$ has $\alpha_{i}$ legs of $b_{i}$ to its left and $\beta_{i}$ legs of $b_{i}$ to its right. We then conclude $\left(P_{p} \otimes P_{q}\right) T_{r} \neq 0$ similarly to (b).

(d) Put $N:=1$ and $k_{0}:=k(\mathcal{C})>0$. We find a partition $p \in \mathcal{C}$ such that $c(p)=k_{0}$, by definition of $k(\mathcal{C})$. Using rotation and [36, Lemma 2.6(d)], we may assume that $p$ consists only of lower points, hence $p \in P^{\bullet \bullet}(0, m)$ for some $m \geqslant k_{0}$. Then $m=c_{\circ}(p)+c_{\bullet}(p)$ and $k_{0}=c(p)=c_{\circ}(p)-c_{\bullet}(p)$, thus $p$ has $\frac{m+k_{0}}{2}$ white points and $\frac{m-k_{0}}{2}$ black points. 
Using [36, Lemma 1.1(b)], we may erase $\frac{m-k_{0}}{2}$ pairs of a white and a black point and we obtain a partition $p_{0} \in \mathcal{C}^{\text {white }}\left(0, k_{0}\right)$ on $k_{0}$ white points. Put $r:=$ ? $\otimes p_{0}^{*} \in \mathcal{C}^{\text {white }}\left(1+k_{0}, 1\right)$. It satisfies $r r^{*}=\varrho^{\otimes N}$ for $N=1$.

Moreover, $\mathcal{C}^{\text {white }}(l, l+t)=\varnothing$ for $0<t<k(\mathcal{C})$, since if we had $p \in \mathcal{C}^{\text {white }}(l, l+t)$, then $c(p)=t$, but $c(p)$ is an integer multiple of $k(\mathcal{C})$ by [36, Proposition 2.7].

\section{Example 3.8.}

(a) If $\mathbb{G}$ is free orthogonal easy $(u=\bar{u})$ with category $\mathcal{C} \subset N C^{\circ}$, then $k(\mathcal{C})=1$ if $\uparrow \in \mathcal{C}$ and $k(\mathcal{C})=2$ otherwise, see [36, Section 7]. Moreover, as $\bullet$ is in $\mathcal{C}$, we also have $\square_{0} \otimes \bullet \in \mathcal{C}$. Hence, conditions $\left(\mathrm{C}_{P} 1\right)$ and $\left(\mathrm{C}_{P} 2\right)$ hold for $S_{n}^{+}, O_{n}^{+}$and the other five free orthogonal easy quantum groups.

(b) For $U_{n}^{+}$, we have $k(\mathcal{C})=0$, thus conditions $\left(\mathrm{C}_{P} 1\right)$ and $\left(\mathrm{C}_{P} 2\right)$ are violated.

(c) The quantum reflection groups $H_{n}^{s+}$ of Example 2.3 have the parameter $k(\mathcal{C})=s$ and o०ø $\in \mathcal{C}$, thus conditions $\left(\mathrm{C}_{P} 1\right)$ and $\left(\mathrm{C}_{P} 2\right)$ hold.

Theorem 3.7 is about as far as we can go for free easy quantum groups in general: indeed, if Theorem 3.7 together with Proposition 3.4 provide a way to prove that some fixed point algebras are Kirchberg algebras, the exact identification of the fixed point algebra requires a computation of $K$-theory that can only be performed for definite fusion rules. For this reason, we focus on examples in the rest of the paper.

\subsection{Free actions}

In this subsection, we investigate the relation between our fixed point construction and free actions. Free actions appear in articles such as $[7,13,14]$. However, there are not that many concrete examples available, and for this reason we prove that our construction generates new examples.

We remind the reader of the following Definition 2.4 of [17] (see also [14]):

Definition 3.9. Given a CQG $\mathbb{G}$, an action $\underline{\delta}: A \rightarrow A \otimes C(\mathbb{G})$ on a $C^{*}$-algebra $A$ is called free if $(A \otimes 1) \underline{\delta}(A)$ is dense in $A \otimes C(\mathbb{G})$.

In order to discuss more easily the above definition, we follow the terminology and notations of $[7,13,21]$ and introduce the canonical map can: $A \otimes A \rightarrow A \otimes C(\mathbb{G})$ given by $\operatorname{can}\left(a \otimes a^{\prime}\right):=$ $(a \otimes 1) \underline{\delta}\left(a^{\prime}\right)$. The condition above is therefore that the map can has a dense image.

Remark 3.10. The $\left(C^{*}\right.$-)freeness property defined above has strong ties with the notion of Hopf-Galois extension (first defined in [26] - see for instance [28]). Indeed, Woronowicz proved [44] that any CQG contains a canonical dense Hopf $*$-algebra $\mathcal{O}(\mathbb{G})$. Using the action of $\mathbb{G}$ on $A$, we can in turn define the Peter-Weyl subalgebra $\mathcal{P}_{\mathbb{G}}(A)$ of $A$ (see, e.g., [7]) - which is not a $C^{*}$-algebra but just a $*$-algebra. The preprint $[7, \mathrm{p} .3]$ proves in its Theorem 0.4 that the action $\underline{\delta}$ is $\left(C^{*}\right.$-)free if and only if $\mathcal{P}_{\mathbb{G}}(A)$ is a Hopf-Galois extension over its fixed point algebra - compare the Peter-Weyl-Galois condition of [7, Definition 0.2, p. 3] with Definition 2.2 of Hopf-Galois extensions in [28, p. 372].

Going back to our initial motivations concerning free actions, we prove:

Proposition 3.11. Let $\mathbb{G}$ be a compact matrix quantum group and $u$ its fundamental representation. If $\mathbb{G}$ satisfies condition $(\mathrm{C} 1)$ then the action $\underline{\delta}$ induced from Proposition 2.4 is free. 
Remark 3.12. At this point, it may seem plausible that condition $(\mathrm{C} 1)$ is equivalent to freeness of the action of $\mathbb{G}$ on $\mathcal{O}_{n}$. However, as we have seen in [19, Notation 3.1], (C1) is not satisfied for the natural representation of $\mathbb{G}=\mathrm{U}(1)$ (or multiple thereof). Using the same kind of argument as in the proof below, it appears that the action of $\mathrm{U}(1)$ on $\mathcal{O}_{2}$ is free.

Of course, this action is not strictly induced from $U(1)$ by Proposition 2.4. But we can build on this example by considering $\mathrm{SU}(2) \times \mathrm{U}(1)$. This is a CMQG which does not satisfy condition $(\mathrm{C} 1)$, but which still acts freely on $\mathcal{O}_{2}$.

Proof of Proposition 3.11. For our proof, we will consider the set

$$
\mathcal{S}:=\left\{s \in C(\mathbb{G}) \mid \exists k_{m} \in \mathbb{N}_{0}, v_{m}, w_{m} \in \mathscr{H}^{\otimes k_{m}}, \operatorname{can}\left(\sum_{m}^{\text {finite }} v_{m}^{*} \otimes w_{m}\right)=1 \otimes s\right\} .
$$

It is clear from the definition of $\underline{\delta}$ that for any $i, j$,

$$
\operatorname{can}\left(S_{j}^{*} \otimes S_{i}\right)=\left(S_{j}^{*} \otimes 1\right)\left(\sum_{k=1}^{n} S_{k} \otimes u_{k i}\right)=1 \otimes u_{j i} .
$$

This means that $\mathcal{S}$ contains all $u_{j i}$. Now, if $v_{i, m}, w_{i, m}$ are elements in $\mathscr{H}^{\otimes k_{i}}$ for $i=1,2$ and such that $\operatorname{can}\left(\sum_{m} v_{i, m}^{*} \otimes w_{i, m}\right)=1 \otimes s_{i}$, then

$$
\begin{aligned}
\operatorname{can}\left(\sum_{m, l} v_{2, m}^{*} v_{1, l}^{*} \otimes w_{1, l} w_{2, m}\right) & =\left(\sum_{m, l} v_{2, m}^{*} v_{1, l}^{*} \otimes 1\right) \underline{\delta}\left(w_{1, l} w_{2, m}\right) \\
& =\left(\sum_{m} v_{2, m}^{*} \otimes 1\right)\left(\sum_{l} v_{1, l}^{*} \otimes 1\right) \underline{\delta}\left(w_{1, l}\right) \underline{\delta}\left(w_{2, m}\right) \\
& =\left(\sum_{m} v_{2, m}^{*} \otimes 1\right)\left(1 \otimes s_{1}\right) \underline{\delta}\left(w_{2, m}\right) \\
& =\left(1 \otimes s_{1}\right)\left(\sum_{m} v_{2, m}^{*} \otimes 1\right) \underline{\delta}\left(w_{2, m}\right) \\
& =\left(1 \otimes s_{1}\right)\left(1 \otimes s_{2}\right)=1 \otimes s_{1} s_{2} .
\end{aligned}
$$

This in turn proves that $\mathcal{S}$ is stable under multiplication. It follows that $\mathcal{S}$ is an algebra, which contains all polynomials in $u_{i j}$.

If the generators of $\mathbb{G}$ are selfadjoint, the polynomials in $u_{i j}$ coincide with the $*$-polynomials in $u_{i j}$ and they are all contained in $\mathcal{S}$, thus $\mathcal{S}$ is dense in $C(\mathbb{G})$.

In general, we can always decompose $u$ into irreducible representations, which all correspond to unitary matrices with coefficients in $C(\mathbb{G})$. Up to a conjugation by a scalar-valued unitary matrix, the algebra generated by the entries of $u$ and their adjoints is the same as the algebra generated by the irreducible components and their adjoints. So without loss of generality, we assume that $u$ is an irreducible representation. It is well known (see, e.g., [30, Definition 1.3.8, p. 11]) that the contragredient of $\left(u_{i j}\right)$ is $\left(u_{i j}^{*}\right)$ in a suitable basis. $u$ is irreducible, so condition (C1) implies that up to equivalence, we can recover the coefficients $\left(u_{i j}^{*}\right)$ through a subrepresentation of a high enough power $\mathscr{H}^{\otimes k}$. It follows that $\mathcal{S}$ contains the $*$-polynomials in $u_{i j}$ and thus is dense in $C(\mathbb{G})$.

Given any $T \in \mathcal{O}_{n}$, for any can $\left(\sum v_{j}^{*} \otimes w_{j}\right)=1 \otimes s$ we have

$$
\operatorname{can}\left(\sum T v_{j}^{*} \otimes w_{j}\right)=(T \otimes 1) \operatorname{can}\left(\sum v_{j}^{*} \otimes w_{j}\right)=T \otimes s .
$$

It follows that the image of can is dense in $\mathcal{O}_{n} \otimes C(\mathbb{G})$ and thus the action $\underline{\delta}$ is free. 
Remark 3.13. On the one hand, it follows from the proof above that assumption (C1) is not needed for Proposition 3.11 in the orthogonal case (i.e., if all the entries of the fundamental representation are selfadjoint). On the other hand, the argument above also shows that in this orthogonal case condition (C1) is automatically satisfied.

Theorem 3.14. The actions of $S_{n}^{+}, \mathrm{O}_{n}^{+}$and $\mathrm{H}_{n}^{s+}$ are free.

\section{Examples $-O_{n}^{+}$and $S_{n}^{+}$}

To illustrate our previous results, we consider three cases, namely those of $O_{n}^{+}, S_{n}^{+}$and of the quantum reflection groups $H_{n}^{s+}$ - which we are going to consider separately in the next section.

We start with the case of $O_{n}^{+}$. This free easy quantum group was introduced by $\mathrm{S}$. Wang in [40] and its fusion rules were studied by Banica in [1]. It appears that it shares the same fusion rules as $\mathrm{SU}(2)$, i.e., its irreducible representations are denoted by $u_{k}$ for $k \in \mathbb{N}_{0}-u_{0}$ being the trivial representation - and the tensor products decompose into

$$
u_{k} \otimes u_{l}=u_{|k-l|} \oplus u_{|k-l|+2} \oplus \cdots \oplus u_{k+l} .
$$

It is clear from the statement of conditions $(\mathrm{C} 1)$ and $(\mathrm{C} 2)$ that they only depend on the fusion rules of the quantum group and not on the quantum group itself. This provides an elementary way to recover the result of Example 3.8(a) in this case. In [19], the case of $\mathrm{SU}_{q}(2)$ was studied in detail (see Section 7.1, p. 17, therein). The computation of the $K$-theory of the fixed point algebra also depends only on the fusion rules. Since the fundamental representation of $\mathrm{O}_{n}^{+}$ corresponds to the natural representation of $\mathrm{SU}(2)$, the proof of Proposition 7.10 [19, p. 1031] applies verbatim and we get Theorem 4.1 below for $\mathbb{G}=O_{n}^{+}$.

The case of $S_{n}^{+}$is very similar: this CMQG was introduced by S. Wang in [39] and its fusion rules were computed by Banica in [2]. The fusion rules are the same as those of $\mathrm{SO}(3)$, i.e., we take the fusion rules (4.1) of $\mathrm{SU}(2)$ but consider only even representations $u_{2 k}, k \in \mathbb{N}_{0}$. By Example 3.8(a), conditions $(\mathrm{C} 1)$ and $(\mathrm{C} 2)$ are satisfied. The fundamental representation $u$ of $S_{n}^{+}$decomposes into $u=u_{0} \oplus u_{2}=u_{1} \otimes u_{1}$ and this shows that the proof of Proposition 7.10 of [19] applies again:

Theorem 4.1. For $\mathbb{G}=O_{n}^{+}$and $\mathbb{G}=S_{n}^{+}$, the fixed point algebra $\mathcal{O}^{\alpha}$ obtained from Proposition 2.4 via the fundamental representation $u$ is a Kirchberg algebra in the UCT class $\mathscr{N}$ whose $K$-theory is

$$
K_{0}\left(\mathcal{O}^{\alpha}\right)=\mathbb{Z}, \quad K_{1}\left(\mathcal{O}^{\alpha}\right)=0 .
$$

Moreover, $\left[1_{\mathcal{O}^{\alpha}}\right]_{0}=1$ and therefore $\mathcal{O}^{\alpha}$ is $C^{*}$-isomorphic to the infinite Cuntz algebra $\mathcal{O}_{\infty}$.

\section{$5 \quad$ Examples - quantum reflection groups}

As mentioned in Example 2.3(d), the quantum reflection groups $H_{n}^{s+}$ were studied by Banica, Belinschi, Capitaine and Collins in [3]. Their fusion rules were computed by Banica and Vergnioux in their article [6] - see in particular Theorem 7.3, p. 348, therein. We follow the notations used in their article up to a point: for a reason that will be clear later on, we write $r_{s}$ for the representation denoted by $r_{0}$ in the original article. For the reader's convenience, we reproduce here the fusion rules of these quantum groups, as described in [6, Theorem 7.3]. The monoid $F=\langle\mathbb{Z} / s \mathbb{Z}\rangle$ of words over $\mathbb{Z} / s \mathbb{Z}$ is equipped with an involution and a fusion operation:

(1) Involution: $\left(i_{1} \ldots i_{k}\right)^{-}=\left(-i_{k}\right) \cdots\left(-i_{1}\right)$.

(2) Fusion: $\left(i_{1} \ldots i_{k}\right) \cdot\left(j_{1} \ldots j_{l}\right)=i_{1} \ldots i_{k-1}\left(i_{k}+j_{1}\right) j_{2} \ldots j_{l}$. 
Using these relations, the fusion rules can be written

$$
r_{x} \otimes r_{y}=\sum_{x=v z, y=\bar{z} w} r_{v w}+r_{v \cdot w}
$$

where $v \cdot w$ is not defined when $v$ or $w$ is the empty word. In the case $z=\varnothing$ ("leading terms of the fusion rule"), we distinguish between the term $r_{x y}$ - that we call the concatenation term and the term $r_{x \cdot y}$ that we call the product term. We are going to compute the $K$-theory of the fixed point algebra generated from the natural representation (indexed by $r_{1}$ ).

In the lemma below, we gather useful results:

Lemma 5.1. For any quantum reflection group $\mathbb{G}=H_{n}^{s+}$,

- for all $0<\ell \leqslant s$, the representation $r_{\ell}$ appears in $\left(r_{1}\right)^{\ell}$, i.e., $r_{\ell} \leqslant\left(r_{1}\right)^{\ell}$;

- moreover, $r_{s-1}$ and $r_{1}$ are contragredient to one another, i.e., $1 \leqslant\left(r_{1}\right)^{s}$;

- all irreducible representations of $\mathbb{G}$ appear as irreducible components of some $r_{1}^{\ell}$ for $\ell$ large enough. Actually,

$$
r_{\sigma_{1} \ldots \sigma_{k}} \leqslant\left(r_{1}\right)^{\sigma_{1}+\cdots+\sigma_{k}}
$$

Remark 5.2. It follows immediately from this lemma together with Woronowicz's abstract existence of a contragredient for any irreducible representation that condition (C1) is satisfied for $H_{n}^{s+}$ and its representation $r_{1}$.

Proof of Lemma 5.1. We prove the first point by induction on $\ell$ : the result is true and obvious for $\ell=1$. For $\ell=2$,

- if $s=2$ then $r_{1} \cdot r_{1}=r_{11}+r_{2}+1$ and the result is true;

- if $s>2$, then $r_{1} \cdot r_{1}=r_{11}+r_{2}$, i.e., $r_{2} \leqslant\left(r_{1}\right)^{2}$ and thus the property is true for $\ell=2$.

Let us now assume the property for $\ell>1$, then (provided $\ell+1<s), r_{\ell} \cdot r_{1}=r_{\ell 1}+r_{\ell+1} \leqslant\left(r_{1}\right)^{\ell+1}$, which shows the result for $\ell+1$.

If $\ell+1=s$, then the product becomes

$$
r_{\ell} \cdot r_{1}=r_{\ell 1}+r_{\ell+1}+1
$$

The first two terms above correspond to $z=\varnothing$, while the third one corresponds to $z=\ell$, i.e., $\bar{z}=-\ell=1$ (equality in $\mathbb{Z} / s \mathbb{Z}$ ).

Let $r_{\sigma_{1} \ldots \sigma_{k}}$ be any irreducible representation in $H_{n}^{s+}$ (where all $\sigma_{j}$ are taken between 1 and $s$ ), then it appears as an irreducible component of $r_{1}^{\sigma_{1}+\cdots+\sigma_{k}}$ : our first point proved that $r_{\sigma}$ appears in $r_{1}^{\sigma}$. Therefore, in the decomposition into irreducible components of $r_{1}^{\sigma_{1}+\cdots+\sigma_{k}}=$ $\left(r_{1}\right)^{\sigma_{1}} \cdots\left(r_{1}\right)^{\sigma_{k}}$, there appears a product of $r_{\sigma_{1}} \cdot r_{\sigma_{2}} \cdots r_{\sigma_{k}}$, which in turn produces a copy of $r_{\sigma_{1} \ldots \sigma_{k}}$ - by using iteratively only the first (concatenation) term of the fusion rules, for $z=\varnothing$.

This lemma enables us to set:

Definition 5.3 (degree function for $H_{n}^{s+}$ ). Given an irreducible representation $p$ of $\mathbb{G}$, we denote by $\delta(p)$ the smallest integer $\ell$ such $p \leqslant r_{1}^{\ell}$.

We can actually give an explicit estimate of $\delta$ :

Proposition 5.4. The degree of the irreducible representation $r_{\sigma_{1} \ldots \sigma_{k}}$, where the $\sigma_{j}$ s are chosen with $0<\sigma_{j} \leqslant s$, is $\delta\left(r_{\sigma_{1} \ldots \sigma_{k}}\right)=\sigma_{1}+\cdots+\sigma_{k}$. 
Proof. Given any irreducible representation $r_{x_{1} \ldots x_{k}}$ with for all $i, 0<x_{i} \leqslant s$, we define $\sigma\left(r_{x_{1} \ldots x_{k}}\right)=x_{1}+\cdots+x_{k}$. It follows by direct examination of (5.1) that if $\gamma \leqslant \alpha \cdot \beta$, then

$$
\sigma(\gamma) \leqslant \sigma(\alpha)+\sigma(\beta)
$$

Iterating the argument and combining it with (5.2), it appears that for all irreducible representation, $\gamma \leqslant\left(r_{1}\right)^{\sigma(\gamma)}$, i.e., $\delta(\gamma) \leqslant \sigma(\gamma)$.

Conversely, if $\gamma \leqslant\left(r_{1}\right)^{\ell}$, then iterating the "subadditivity property" (5.3) of $\sigma$ and using $\sigma\left(r_{1}\right)=1$, we get: $\sigma(\gamma) \leqslant \ell$. Since this is valid for all $\ell$, we get $\sigma(\gamma) \leqslant \delta(\ell)$. This proves that $\delta(\gamma)=\sigma(\gamma)$ for all irreducible $\gamma$ and concludes the proof.

Lemma 5.5. For all irreducible representations $\alpha=r_{x}, \beta=r_{y}$ and $\gamma$ with $\gamma \leqslant \alpha \cdot \beta$,

$$
\delta(\gamma) \leqslant \delta(\alpha)+\delta(\beta)
$$

For $\mathbb{G}=H_{n}^{s+}$, the cases of equality in (5.4) can only occur for the terms $z=\varnothing$ of (5.1). For those terms, the equality is true unconditionally for $r_{x y}$, and only if $x_{k}+y_{1} \leqslant s$ for $r_{x \cdot y}$.

Remark 5.6. The above lemma is the reason why we use the notation $r_{s}$ instead of $r_{0}$.

Proof of Lemma 5.5. The inequality (5.4) follows from

$$
\gamma \leqslant \alpha \cdot \beta \leqslant\left(r_{1}\right)^{\delta(\alpha)}\left(r_{1}\right)^{\delta(\beta)}=\left(r_{1}\right)^{\delta(\alpha)+\delta(\beta)} .
$$

This is just a variation on the proof of Lemma 5.1 above.

The equality requires to study the behavior of the total degree in the fusion rules, starting from two irreducible representations $\alpha=r_{x}=r_{x_{1} \ldots x_{k}}$ and $\beta=r_{y}=r_{y_{1} \ldots y_{l}}$ with finite sequences $\left(x_{i}\right)$ and $\left(y_{j}\right)$ taking their values in $\{1, \ldots, s\}$.

If $\gamma$ arises from a term in (5.1) with $z \neq \varnothing$, then the inequality (5.4) is strict. Indeed, $\gamma$ could then arise from the $z=\varnothing$ term of $v$ and $w$, where $x=v z$ and $y=\bar{z} w$.

Assuming now that $z=\varnothing$, using the estimate of the degree of Proposition 5.4, the term $r_{x_{1} \ldots x_{k} y_{1} \ldots y_{l}}$ yields an equality case for (5.4). The same is true of the term $r_{x_{1} \ldots\left(x_{k}+y_{1}\right) \ldots y_{l}}$ provided $x_{k}+y_{1} \leqslant s$. It remains to treat the case of $x_{k}+y_{1}>s$, but such a term corresponds to a strict inequality in (5.4) and this completes the proof.

We will use the following notations extensively: let $R_{\ell}$ (resp. $\partial R_{\ell}$ ) be the $\mathbb{Z}$-free module constructed on irreducible representations appearing in $\left(r_{1}\right)^{\ell}$ (resp. appearing in $\left(r_{1}\right)^{\ell}$ and not in any $\left(r_{1}\right)^{k}$ for $0 \leqslant k<\ell$ ).

It follows immediately from the definition of $R_{\ell}$ that $R_{\ell} \cdot R_{k} \subseteq R_{\ell+k}$, where the product $R_{\ell} \cdot R_{k}$ is taking place in the fusion ring of $\mathbb{G}$.

Lemma 5.7. The fusion rules (5.1) actually ensure that in $\mathbb{Z} / s \mathbb{Z}$ :

$$
[\delta(\gamma)]=[\delta(\alpha)]+[\delta(\beta)]
$$

Proof. Direct examination and Proposition 5.4 show that if $z=\varnothing$, then $r_{x y}=r_{i_{1} \ldots i_{k} j_{1} \ldots j_{l}}$ has degree $\delta\left(r_{x y}\right)=i_{1}+\cdots+i_{k}+j_{1}+\cdots+j_{l}=\delta\left(r_{x}\right)+\delta\left(r_{y}\right)$. The degree of $r_{x \cdot y}$ is the same in $\mathbb{Z} / s \mathbb{Z}$ (since a simplification in $\mathbb{Z} / s \mathbb{Z}$ may happen in the fusion $x \cdot y$ ).

More generally, if $z \neq \varnothing$, then the definition of the involution on the monoid $F$ ensures that taking out both $z$ and $\bar{z}$ do not change $\left[\delta\left(r_{v w}\right)\right]$ in $\mathbb{Z} / s \mathbb{Z}$. However, simplifying by $z$ and $\bar{z}$ lessen the total degree of the expression, i.e., the degree $\delta\left(r_{v w}\right)$ has to be strictly less than $\delta\left(r_{x}\right)+\delta\left(r_{y}\right)$. The same argument applies to $r_{v+w}$.

We are now in position to compute the chain group $\mathfrak{C}(\mathbb{G})$ as introduced in $[8,29]$. This object is also known as universal grading group, see, e.g., [20]. 
Proposition 5.8. The chain group of $\mathbb{G}=H_{n}^{s+}$ is $\mathfrak{C}(\mathbb{G})=\mathbb{Z} / s \mathbb{Z}$.

Proof. The equation (5.5) shows that if $\tau_{1}, \ldots, \tau_{k}, p, q$ are irreducible representations and that they satisfy $p, q \leqslant \tau_{1} \cdot \tau_{2} \cdots \tau_{k}$ (both $p$ and $q$ appear in the fusion product), then (in the group $\mathbb{Z} / s \mathbb{Z})$

$$
[\delta(p)]=\left[\delta\left(\tau_{1}\right)\right]+\cdots+\left[\delta\left(\tau_{k}\right)\right]=[\delta(q)] .
$$

In other words, if $p$ and $q$ have the same class in the chain group $\mathfrak{C}(\mathbb{G})$, then $[\delta(p)]=[\delta(q)]$.

Conversely, take $[\delta(p)]=[\delta(q)]$ in $\mathbb{Z} / s \mathbb{Z}$. If $\delta(p)=\delta(q)=\ell$, then both representations appear in $\left(r_{1}\right)^{\ell}$ and they have the same class in the chain group. Otherwise, without loss of generality, we can assume that $\delta(p)>\delta(q)$ and thus there is an integer $k$ such that $\delta(p)=\delta(q)+k s$. By definition of $\delta(q), q \leqslant\left(r_{1}\right)^{\delta(q)}$. We can then use Lemma 5.1 (and especially the part $1 \leqslant\left(r_{1}\right)^{s}$ ) to show

$$
q=q \cdot 1^{k} \leqslant\left(r_{1}\right)^{\delta(q)}\left(r_{1}\right)^{s} \cdots\left(r_{1}\right)^{s}=\left(r_{1}\right)^{\delta(q)+k s} .
$$

This in turn proves that $p$ and $q$ share the same class in $\mathfrak{C}(\mathbb{G})$. The equation (5.5) then ensures that the group law in $\mathfrak{C}(\mathbb{G})$ and $\mathbb{Z} / s \mathbb{Z}$ coincide.

Remark 5.9. It follows from this evaluation of the chain group $\mathfrak{C}(\mathbb{G})$ together with the property $1 \leqslant\left(r_{1}\right)^{s}$ of Lemma 5.1 that condition $(\mathrm{C} 2)$ is satisfied for $\mathbb{G}=H_{n}^{s+}$ equipped with its representation $r_{1}$, for the integers $k_{0}=s$ and $N=1$.

Corollary 5.10. There is a decomposition

$$
R_{\ell}=\bigoplus_{0 \leqslant k \leqslant \ell,[k]=[\ell]} \partial R_{k}
$$

where the equality $[k]=[\ell]$ takes place in $\mathbb{Z} / s \mathbb{Z}$.

Proof. This is a consequence of $p \leqslant\left(r_{1}\right)^{\ell} \Longrightarrow[\delta(p)]=[\ell]$ in $\mathbb{Z} / s \mathbb{Z}$ together with $1 \leqslant\left(r_{1}\right)^{s}$.

Finally, we will need the notion of length $\lambda\left(r_{s}\right)$ of an irreducible representation $r_{s}$, which is just the length (number of letters) of its indexing sequence $s$. It is clear from (5.4) that for all $\gamma \leqslant \alpha \cdot \beta, \lambda(\gamma) \leqslant \lambda(\alpha)+\lambda(\beta)$ with equality only for the concatenation term of $z=\varnothing$.

Theorem 5.11. For $\mathbb{G}=H_{n}^{s+}$ and its representation $\alpha=r_{1}$, condition $(\mathrm{C} 2)$ is satisfied for the integer $k_{0}=s$ and the computation of $K$-theory yields

$$
K_{0}\left(\mathcal{O}^{\alpha}\right)=\bigoplus_{\mathbb{N}} \mathbb{Z}, \quad K_{1}\left(\mathcal{O}^{\alpha}\right)=0
$$

The proof of this theorem is going to require a few intermediate lemmas and a restatement of the problem.

Indeed, Theorem 5.11 above is stated in terms of the representation $\alpha=r_{1}$, but the computations below will be easier if we consider the case of $\alpha=r_{1}^{S}$ - which yields isomorphic results, according to Proposition 5.8 above and [19, Proposition 7.8, p. 1029].

Consider the maps $\varphi: R \rightarrow R$ and $\psi: R \rightarrow R$ given on all $a \in R$ by

$$
\varphi(a)=a\left(r_{1}^{s}-1\right), \quad \psi(a)=a r_{1}^{s} .
$$

The previous properties of degree show that these maps induce $\varphi_{\ell}: R_{\ell} \rightarrow R_{\ell+s}$ and $\psi_{\ell}: R_{\ell} \rightarrow$ $R_{\ell+s}$. The $K$-theory of the fixed point algebra $\mathcal{F}^{\alpha}$ is given by the inductive limit of the system

$$
\cdots \rightarrow R_{\ell} \stackrel{\psi_{\ell}}{\longrightarrow} R_{\ell+s} \stackrel{\psi_{\ell+s}}{\longrightarrow} R_{\ell+2 s} \rightarrow \cdots \text {. }
$$


The general theory presented in [19] (see in particular Theorem 5.4, p. 1025) shows that $K_{0}\left(\mathcal{O}^{\alpha}\right)$ is obtained as the cokernel of the map $\varphi: \lim _{\rightarrow} R_{\ell} \rightarrow \lim _{\rightarrow} R_{\ell}$ defined from the system

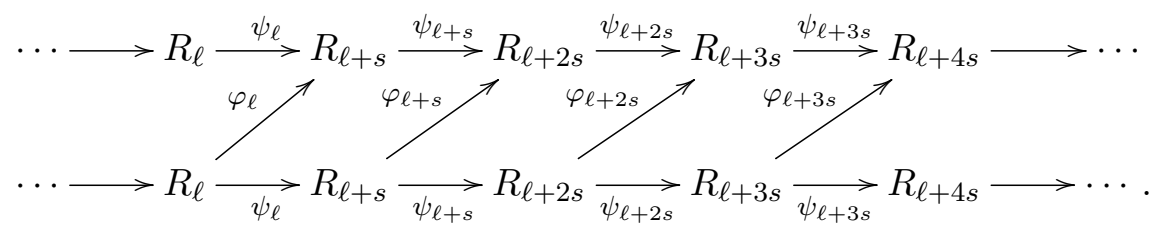

All the squares in the diagram above are commutative - indeed, it amounts to proving that for any $a \in R, a r_{1}^{s}\left(r_{1}^{s}-1\right)=a\left(r_{1}^{s}-1\right) r_{1}^{s}$. Thus, the $\operatorname{map} \varphi: \lim _{\rightarrow} R_{\ell} \rightarrow \lim _{\rightarrow} R_{\ell}$ is well-defined and we can compute its cokernel. To this end, we start by computing the cokernels at each finite level $\ell$ and it is a well-known property that we will obtain the overall cokernel as inductive limit of those finite cokernels.

The evaluation below is the corner stone of our argument:

Lemma 5.12. For any irreducible representation $r_{x}$ with $\delta\left(r_{x}\right)=\ell$, we have

$$
\varphi\left(r_{x}\right)=r_{x}\left(r_{1}^{s}-1\right)=r_{x} \underbrace{1 \ldots 1}_{\text {sterms }}+r_{x s}+m,
$$

where $m$ is a $\mathbb{Z}$-linear combination of irreducible representations in $R_{\ell+s}$ which do not contain any term $r_{x 1 \ldots 1}$ and $r_{x s}$.

Proof. It is clear from the fusion rules that by taking only the concatenation term for $z=\varnothing$ in the $s$ successive fusion products of $r_{x}$ with $r_{1}$, we obtain a term $r_{\mu}=r_{x} \underbrace{1 \ldots 1}_{s \text { terms }}$. Moreover, this irreducible irrepresentation has maximal length (namely $\lambda\left(r_{\mu}\right)=\lambda\left(r_{x}\right)+s$ ) among those appearing in the product $r_{x}\left(r_{1}^{s}-1\right)$. Given $\gamma \leqslant \alpha \cdot \beta$, we know that $\lambda(\gamma) \leqslant \lambda(\alpha)+\lambda(\beta)$ is actually an equality only for the concatenation term of $z=\varnothing$. It follows that there is only one way to obtain a representation of such length. Thus, no further term involving $r_{\mu}$ appear in $\varphi\left(r_{x}\right)$.

For $r_{x s}$, the argument is slightly different: first, we remark that it has maximum degree $\left(\delta\left(r_{x s}\right)=\delta\left(r_{x}\right)+s\right)$. This implies that it was obtained by taking only terms with $z=\varnothing$ in the successive fusion products. We then remark that its length is minimal among those terms obtained by taking only leading terms $(z=\varnothing)$ in the fusion. This in turn ensures that it is (and can only be) obtained by taking product terms in the $s$ successive fusion products. Thus, no further term involving $r_{x s}$ appear in $\varphi\left(r_{x}\right)$.

Lemma 5.13. For all $\ell$, there is free $\mathbb{Z}$-module $C_{\ell} \subseteq R_{\ell}$ such that

$$
R_{\ell+s}=C_{\ell+s} \oplus \varphi_{\ell}\left(R_{\ell}\right) .
$$

Moreover, this free $\mathbb{Z}$-module decomposes according to the degree into

$$
C_{\ell}=\bigoplus_{0 \leqslant k \leqslant \ell,[k]=[\ell]} \partial C_{k}
$$

Remark 5.14. The statement above calls for several remarks:

- The notation $R_{\ell+s}=C_{\ell+s} \oplus \varphi_{\ell}\left(R_{\ell}\right)$ indicates that any element of $R_{\ell+s}$ can be written in a unique way as a sum of an element of $C_{\ell+s}$ and an element of $\varphi_{\ell}\left(R_{\ell}\right)$.

- An immediate consequence of (5.9) is that we can thus identify $C_{\ell+s}$ with the cokernel $R_{\ell+s} / \varphi\left(R_{\ell}\right)$.

- In the decomposition (5.10), we use obvious notations similar to those of (5.6). 
Proof of Lemma 5.13. We proceed by induction: for a minimal level $0 \leqslant \ell<2 s$, the decomposition (5.9) shows that we just have to find $C_{\ell}$ s.t. $R_{\ell}=C_{\ell} \oplus \varphi_{\ell-s}\left(R_{\ell-s}\right)$. Given any $a \in R_{\ell}$, we can use relation (5.8) to cancel any term of the form $r_{x} \underbrace{1 \ldots 1}_{s \text { terms }}$ appearing in $a$. If we then define $C_{\ell}$ as the free $\mathbb{Z}$-module generated on all irreducible representations appearing in $R_{\ell}$ which are not of the form $r_{x} \underbrace{1 \ldots 1}_{s \text { terms }}$, then clearly

$$
R_{\ell}=C_{\ell} \oplus \varphi_{\ell-s}\left(R_{\ell-s}\right) .
$$

Let us now assume that at level $\ell$, we have a decomposition

$$
R_{\ell}=C_{\ell} \oplus \varphi\left(R_{\ell}\right)
$$

where $C_{\ell}=\bigoplus_{0 \leqslant k \leqslant \ell,[k]=[\ell]} \partial C_{k}$, we want to prove that $R_{\ell+s}$ admits a similar decomposition.

A consequence of the decomposition (5.6), is that $\varphi\left(R_{\ell}\right)=\bigoplus_{0 \leqslant k \leqslant \ell,[k]=[\ell]} \varphi\left(\partial R_{k}\right)$.

Let us now introduce $\partial C_{\ell+2}$ as the free $\mathbb{Z}$-module generated by all irreducible representation of degree exactly $\ell+s$ which are not of the form $r_{x} \underbrace{1 \ldots 1}_{s \text { terms }}$, then

$$
R_{\ell+s}=\partial C_{\ell+s} \oplus \varphi\left(\partial R_{\ell}\right) \oplus R_{\ell}=\partial C_{\ell+s} \oplus \bigoplus_{0 \leqslant k \leqslant \ell,[k]=[\ell]} \partial C_{k} \oplus \varphi\left(R_{\ell+s}\right) .
$$

This completes the proof of the existence of the $\mathbb{Z}$-free module $C_{\ell}=\bigoplus_{0 \leqslant k \leqslant \ell,[k]=[\ell]} \partial C_{k}$ which implements the cokernel in $R_{\ell}$.

Let us now study the connecting maps between these cokernels. Remember from the commutation relations appearing in (5.7) that all connecting maps $\psi_{\ell}: R_{\ell} \rightarrow R_{\ell+s}$ induce quotient maps at the level of cokernels, which we denote by

$$
\widetilde{\psi_{\ell}}: R_{\ell} / \varphi\left(R_{\ell-s}\right) \rightarrow R_{\ell+s} / \varphi\left(R_{\ell}\right) .
$$

Lemma 5.15. The connecting maps between the cokernels are the identity: for any $a \in R_{\ell}$, $\widetilde{\psi_{\ell}}\left([a]_{\ell}\right)=[a]_{\ell+s}$, where $[a]_{\ell}$ and $[a]_{\ell+s}$ are the class of a in $R_{\ell} / \varphi\left(R_{\ell-s}\right)$ and $R_{\ell+s} / \varphi\left(R_{\ell}\right)$, respectively.

Proof. Indeed, take any $a \in R_{\ell}$, then $\psi(a)=\varphi(a)+a$. We know that in the cokernel $R_{\ell+s} / \varphi\left(R_{\ell}\right),[\varphi(a)]_{\ell+s}=0$, thus $[\psi(a)]_{\ell+s}=[a]_{\ell+s}$.

Remark 5.16. A consequence of the above Lemma 5.15 is that the inductive $\operatorname{limit} \lim _{\rightarrow} C_{\ell}$ is simply the increasing union of free $\mathbb{Z}$-modules and it suffices to estimate the number of irreducible representations of $H_{n}^{s+}$ of degree $\ell+s$ which are not of the form $r_{x} \underbrace{1 \ldots 1}_{s \text { terms }}$. We do precisely this in the next lemma.

Lemma 5.17. Let $m_{\ell+s}$ be the number of irreducible representations of $H_{n}^{s+}$ of degree $\ell+s$ which are not of the form $r_{x} \underbrace{1 \ldots 1}_{\text {sterms }}$, then $m_{\ell+s} \rightarrow \infty$.

Proof. Let us introduce the number $n_{\ell}$ of irreducible representations of degree exactly $\ell$, then relation (5.8) ensures that

$$
n_{\ell+s} \geqslant 2 n_{\ell} .
$$

Indeed, for each irreducible representation $r_{x}$ of degree $\ell$, there are at least two irreducible representations of degree $\ell+2$, namely $r_{x} \underbrace{1 \ldots 1}_{s \text { terms }}$ and $r_{x s}$. This also forces $m_{\ell+s} \geqslant n_{\ell}$. The relation (5.11), together with the equality $n_{0}=1$, shows that $n_{\ell}$ (and therefore $m_{\ell}$ ) tends to infinity when $\ell$ tends to infinity. 
Proof of Theorem 5.11. It follows from Example 3.8(a) that conditions (C1) and (C2) are satisfied for the fusion rules of the quantum reflection group $\mathbb{G}=H_{n}^{s+}$. Consequently, Proposition 3.4 applies to the fixed point algebra - which is therefore determined up to $*$-isomorphism by its $K$-theory.

To compute $K_{*}\left(\mathcal{O}^{\alpha}\right)$, we use the inductive system (5.7). We first evaluate $K_{1}\left(\mathcal{O}^{\alpha}\right)$ : according to [19, Theorem 5.4 , p. 1025] this $K$-group is the kernel of the map $\varphi$ defined by the inductive system (5.7). If $c$ is a nonzero element in $R$, it can be realised on a finite level $\ell$. Let us consider the top length nonvanishing irreducible representations appearing in $c \in R_{\ell}$ and write $c=$ $\sum \alpha_{j} r_{x_{1}^{j} \ldots x_{\lambda}^{j}}+m$ where $\alpha_{j} \in \mathbb{Z} \backslash\{0\}, \lambda$ is the maximum length of irreducible representations in $c$ and $m$ is a combination of irreducible representations with lower length. Following Lemma 5.12,

$$
\varphi(c)=\sum \alpha_{j} r_{x_{1}^{j} \ldots x_{\lambda}^{j} 1 \ldots 1}+m^{\prime}
$$

where $m^{\prime}$ is a linear combination of irreducible representations which do not contain any term $r_{x_{1}^{j} \ldots x_{\lambda}^{j} 1 \ldots 1}$ (i.e., maximal length terms). It follows that $\varphi(c)-c \neq 0$ (since irreducible representations in $c$ have length at most $\lambda$ and $r_{x_{1}^{j} \ldots x_{\lambda}^{j} 1 \ldots 1}$ has length $\lambda+s$, no cancellation can occur). Essentially the same argument proves that if $c \in R_{\ell}$ is nonvanishing, then $\psi_{\ell}(c) \in R_{\ell+s}$ is also nonvanishing. Consequently, $\operatorname{ker}\left(1-\varphi_{*}\right)=K_{1}\left(\mathcal{O}^{\alpha}\right)=\{0\}$.

The computation of $K_{0}\left(\mathcal{O}^{\alpha}\right)$ is an easy consequence of Lemma 5.13, Remark 5.14 and Lemmas 5.15 and 5.17. The proof of Theorem 5.11 is thus complete.

As a final comment, this paper shows how techniques from classification theory for $C^{*}$ algebras and a thorough understanding of fusion rules can be combined to identify free actions of compact quantum groups on $C^{*}$-algebras. The characterisation of the fixed point algebra requires a concrete computation of $K$-theory, and explains why we restricted ourselves to examples in the second part of the paper. Similar results should however be possible for other classes of CQGs, as soon as we have a fine comprehension of their fusion rules.

\section{Acknowledgements}

The second author was partially funded by the ERC Advanced Grant on Non-Commutative Distributions in Free Probability, held by Roland Speicher, Saarland University. The first author was supported by the Danish National Research Foundation through the Centre for Symmetry and Deformation (DNRF92) and by the Engineering and Physical Sciences Research Council Grant EP/L013916/1, since the first results of this work were obtained during the first author's postdoc in Glasgow.

Both authors are grateful to Roland Speicher's ERC Advanced Grant and Christian Voigt for enabling their respective stays in Scotland where this collaboration started. They also thank the anonymous referees for their thourough reviews and remarks.

\section{References}

[1] Banica T., Théorie des représentations du groupe quantique compact libre $\mathrm{O}(n)$, C. R. Acad. Sci. Paris Sér. I Math. 322 (1996), 241-244, math.QA/9806063.

[2] Banica T., Symmetries of a generic coaction, Math. Ann. 314 (1999), 763-780, math.QA/9811060.

[3] Banica T., Belinschi S.T., Capitaine M., Collins B., Free Bessel laws, Canad. J. Math. 63 (2011), 3-37, arXiv:0710.5931.

[4] Banica T., Bichon J., Collins B., The hyperoctahedral quantum group, J. Ramanujan Math. Soc. 22 (2007), 345-384, math.RT/0701859. 
[5] Banica T., Speicher R., Liberation of orthogonal Lie groups, Adv. Math. 222 (2009), 1461-1501, arXiv:0808.2628.

[6] Banica T., Vergnioux R., Fusion rules for quantum reflection groups, J. Noncommut. Geom. 3 (2009), 327-359, arXiv:0805.4801.

[7] Baum P.F., De Commer K., Hajac P.M., Free actions of compact quantum group on unital $C^{*}$-algebras, arXiv:1304.2812.

[8] Baumgärtel H., Lledó F., Duality of compact groups and Hilbert $C^{*}$-systems for $C^{*}$-algebras with a nontrivial center, Internat. J. Math. 15 (2004), 759-812, math.OA/0311170.

[9] Bichon J., Free wreath product by the quantum permutation group, Algebr. Represent. Theory 7 (2004), 343-362, math.QA/0107029.

[10] Carey A.L., Paolucci A., Zhang R.B., Quantum group actions on the Cuntz algebra, Ann. Henri Poincaré 1 (2000), 1097-1122, q-alg/9705020.

[11] Cuntz J., Simple $C^{*}$-algebras generated by isometries, Comm. Math. Phys. 57 (1977), $173-185$.

[12] Cuntz J., Regular actions of Hopf algebras on the $C^{*}$-algebra generated by a Hilbert space, in Operator Algebras, Mathematical Physics, and Low-Dimensional Topology (Istanbul, 1991), Res. Notes Math., Vol. 5, A K Peters, Wellesley, MA, 1993, 87-100.

[13] Dąbrowski L., Hadfield T., Hajac P.M., Equivariant join and fusion of noncommutative algebras, SIGMA 11 (2015), 082, 7 pages, arXiv:1407.6020.

[14] De Commer K., Yamashita M., A construction of finite index $C^{*}$-algebra inclusions from free actions of compact quantum groups, Publ. Res. Inst. Math. Sci. 49 (2013), 709-735, arXiv:1201.4022.

[15] Doplicher S., Roberts J.E., Duals of compact Lie groups realized in the Cuntz algebras and their actions on $C^{*}$-algebras, J. Funct. Anal. 74 (1987), 96-120.

[16] Doplicher S., Roberts J.E., Endomorphisms of $C^{*}$-algebras, cross products and duality for compact groups, Ann. of Math. 130 (1989), 75-119.

[17] Ellwood D.A., A new characterisation of principal actions, J. Funct. Anal. 173 (2000), 49-60.

[18] Freslon A., Weber M., On the representation theory of partition (easy) quantum groups, J. Reine Angew. Math., to appear, arXiv:1308.6390.

[19] Gabriel O., Fixed points of compact quantum groups actions on Cuntz algebras, Ann. Henri Poincaré 15 (2014), 1013-1036, arXiv:1210.5630.

[20] Gelaki S., Nikshych D., Nilpotent fusion categories, Adv. Math. 217 (2008), 1053-1071, math.QA/0610726.

[21] Hajac P.M., Krähmer U., Matthes R., Zieliński B., Piecewise principal comodule algebras, J. Noncommut. Geom. 5 (2011), 591-614, arXiv:0707.1344.

[22] Kashina Y., Sommerhäuser Y., Zhu Y., On higher Frobenius-Schur indicators, Mem. Amer. Math. Soc. 181 (2006), viii+65 pages, math.RA/0311199.

[23] Kirchberg E., The classification of purely infinite $C^{*}$-algebras using Kasparov theory, Preprint, 1994.

[24] Kirchberg E., Phillips N.C., Embedding of exact $C^{*}$-algebras in the Cuntz algebra $\mathcal{O}_{2}$, J. Reine Angew. Math. 525 (2000), 17-53, funct-an/9712002.

[25] Konishi Y., Nagisa M., Watatani Y., Some remarks on actions of compact matrix quantum groups on $C^{*}$-algebras, Pacific J. Math. 153 (1992), 119-127.

[26] Kreimer H.F., Takeuchi M., Hopf algebras and Galois extensions of an algebra, Indiana Univ. Math. J. 30 (1981), 675-692.

[27] Marciniak M., Actions of compact quantum groups on $C^{*}$-algebras, Proc. Amer. Math. Soc. 126 (1998), 607-616.

[28] Montgomery S., Hopf Galois theory: a survey, in New Topological Contexts for Galois Theory and Algebraic Geometry (BIRS 2008), Geom. Topol. Monogr., Vol. 16, Geom. Topol. Publ., Coventry, 2009, 367-400.

[29] Müger M., On the center of a compact group, Int. Math. Res. Not. 2004 (2004), 2751-2756.

[30] Neshveyev S., Tuset L., Compact quantum groups and their representation categories, Cours Spécialisés, Vol. 20, Société Mathématique de France, Paris, 2013, available at http://folk.uio.no/sergeyn/papers/ CQGRC.pdf.

[31] Paolucci A., Coactions of Hopf algebras on Cuntz algebras and their fixed point algebras, Proc. Amer. Math. Soc. 125 (1997), 1033-1042. 
[32] Pinzari C., Simple $C^{*}$-algebras associated with compact groups and K-theory, J. Funct. Anal. 123 (1994), $46-58$.

[33] Pinzari C., Roberts J.E., A duality theorem for ergodic actions of compact quantum groups on $C^{*}$-algebras, Comm. Math. Phys. 277 (2008), 385-421, math.OA/0607188.

[34] Pinzari C., Roberts J.E., A rigidity result for extensions of braided tensor $C^{*}$-categories derived from compact matrix quantum groups, Comm. Math. Phys. 306 (2011), 647-662, arXiv:1007.4480.

[35] Rørdam M., Classification of nuclear, simple $C^{*}$-algebras, in Classification of Nuclear $C^{*}$-algebras. Entropy in Operator Algebras, Encyclopaedia Math. Sci., Vol. 126, Springer, Berlin, 2002, 1-145.

[36] Tarrago P., Weber M., The classification of tensor categories of two-colored noncrossing partitions, arXiv:1509.00988.

[37] Tarrago P., Weber M., Unitary easy quantum groups: the free case and the group case, Int. Math. Res. Not., to appear, arXiv:1512.00195.

[38] Tikuisis A., White S., Winter W., Quasidiagonality of nuclear $C^{*}$-algebras, Ann. of Math., to appear, arXiv:1509.08318.

[39] Wang S., Free products of compact quantum groups, Comm. Math. Phys. 167 (1995), 671-692.

[40] Wang S., Quantum symmetry groups of finite spaces, Comm. Math. Phys. 195 (1998), 195-211, math.OA/9807091.

[41] Woronowicz S.L., Compact matrix pseudogroups, Comm. Math. Phys. 111 (1987), 613-665.

[42] Woronowicz S.L., Twisted SU(2) group. An example of a noncommutative differential calculus, Publ. Res. Inst. Math. Sci. 23 (1987), 117-181.

[43] Woronowicz S.L., Tannaka-Krein duality for compact matrix pseudogroups. Twisted SU(N) groups, Invent. Math. 93 (1988), 35-76.

[44] Woronowicz S.L., Compact quantum groups, in Symétries Quantiques (Les Houches, 1995), North-Holland, Amsterdam, 1998, 845-884. 